\section{TD-02 KIDNEY TISSUE DAMAGE IN MICE WITH SINGLE AND COMBINED ABNORMALITIES IN COMPLEMENT, INTERFERON AND APOPTOTIC CELL CLEARANCE}

${ }^{1}$ Sladjana Skopelja-Gardner, 'YuFeng Peng, 'Lucrezia Colonna, 'Xizhang Sun, ${ }^{1}$ Lena Tanaka, ${ }^{2}$ Anne Davidson, ${ }^{3}$ David I Salant, ${ }^{1,4}$ Keith B Elkon*. ${ }^{1}$ Division of Rheumatology, University of Washington, Seattle, Washington, USA; ${ }^{2}$ Center for Autoimmune and Musculoskeletal Diseases, The Feinstein Institute for Medical Research, New York, NY, USA; ${ }^{3}$ Division of Nephrology, Boston University, Boston, MA, USA; ${ }^{4}$ Department of Immunology, University of Washington, Seattle, Washington, USA

\subsection{6/lupus-2018-Ism.121}

Background Lupus nephritis (LN) affects $\sim 70 \%$ of systemic lupus erythematosus (SLE) patients and is one of the main contributors to morbidity and mortality. While defective clearance of apoptotic cells (AC), immune complexes, and type 1 interferons (IFN) are strongly implicated in lupus pathogenesis, the precise way that each impacts kidney protection and injury is unknown.

Methods To investigate mechanisms of kidney injury in a lupus-like disease model, we created $\mathrm{C} 57 \mathrm{BL} / 6$ mice with defective clearance of AC (Mfge8-/-) and anti-chromatin antibodies (sle1) that were also deficient in either C1q [C1q Triple mutant (C1qTM)] or C3 (C3TM). Kidney injury was evaluated by urine albumin/creatinine ratio (UCAR), PAS staining, and immunofluorescence (IF) staining. The effect of IFN-I on disease was studied in C3TM mice by a single injection of an adenovirus expressing IFN $\alpha(\mathrm{AdV}$-IFN $\alpha)$.

Results Sle1 mice deficient in MFGE8 developed significantly higher titers of autoantibodies directed at lupus antigens compared to sle1 mice alone. When MFGE8-/- Sle1 mice also had $\mathrm{C} 1 \mathrm{q}$ or $\mathrm{C} 3$ deficiency, a further increase in anti-DNA (figure 1A) and other autoantibodies was observed. Both TM strains showed AC accumulation in the kidneys (figure 1B) and C1qTM mice had decreased survival. Remarkably, we detected glomerular deposition of $\mathrm{C} 3 / \mathrm{C} 3 \mathrm{~d}$ in $\mathrm{C} 1 \mathrm{qTM}$ (figure 1C) and the membrane attack complex (MAC) in C3TM mice. To dissociate the effects of complement on $\mathrm{B}$ cells versus effects on the kidney, we studied antibody mediated kidney injury (Nephrotoxic Nephritis, NTN) in mice deficient in AC clearance and complement proteins [double knockout (DKO)
(Mfge8-/-C1q-/- or Mfge8-/-C3-/-) mice]. NTN in C1q DKO and C3 DKO mice revealed a significantly elevated UACR compared to the single mutants. IF analyses also revealed glomerular $\mathrm{C} 3 / \mathrm{C} 3 \mathrm{~d}$ deposition in $\mathrm{C} 1 \mathrm{qDKO}$ mice and MAC deposition in C3DKO mice. A single injection of AdV-IFN $\alpha$ accelerated kidney damage in C3TM mice, resulting in increased anti-dsDNA IgG titers, UACR, and PAS staining.

Conclusions These findings demonstrate that early component complement deficiencies have two distinct effects: they promote enhanced B cell autoreactivity and they protect against kidney disease. Increased glomerular C3/C3d deposition in C1qTM and NTN C1qDKO mice suggest activation of the lectin or alternative complement pathways. Increased MAC deposition in C3TM and NTN C3DKO mice indicates that a C3-independent mechanism leads to distal complement activation and MAC formation. These data prompt models of tissue injury in low complement states that will require assessment in human SLE and provide rationale for targeted therapeutics that are not currently used.

\section{TD-03 CONVOLUTIONAL NEURAL NETWORKS IDENTIFY IN SITU ADAPTIVE IMMUNE CELL ARCHITECTURES IN HUMAN} LUPUS

${ }^{1}$ Vladimir M Liarski, ${ }^{1}$ Adam Sibley, ${ }^{2}$ Nicholas van Panhuys, 'Lily Junting, ${ }^{1}$ Anthony Chang, ${ }^{1}$ Domenick Kennedy, ${ }^{1}$ Maria Merolle, ${ }^{2}$ Ron Germain, 'Maryellen Giger, ${ }^{1}$ Marcus R Clark*. ${ }^{1}$ The University of Chicago; ${ }^{2}$ National Institutes of Health

\subsection{6/lupus-2018-Ism.122}

Background Adaptive immunity is driven by antigen-restricted cell:cell interactions. In mice, two-photon excitation microscopy (TPEM) has revolutionized our understanding of immune cell architectures. However, TPEM has several limitations: most notably it can only be used to study manipulated animal model systems and not human disease. Previously, we demonstrated that by quantifying the distance between B cells and $\mathrm{T}$ cells in multichannel confocal images of human tissue (Cell Distance Mapping, CDM) we could identify cognate interactions. However, CDM used fixed filters and could not

A
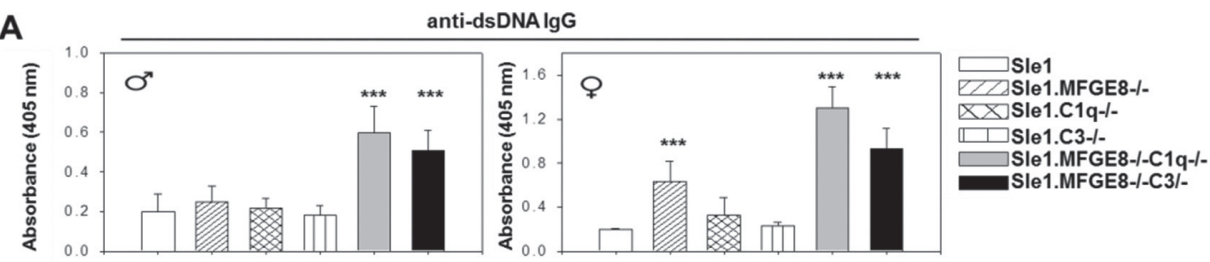

B

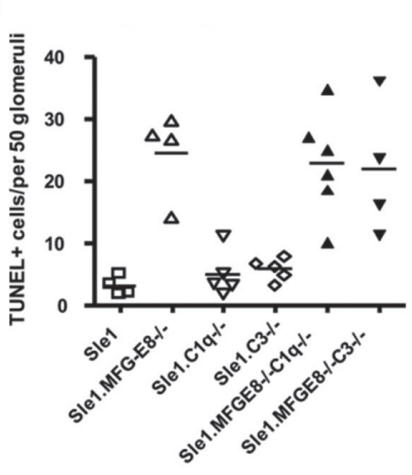

C

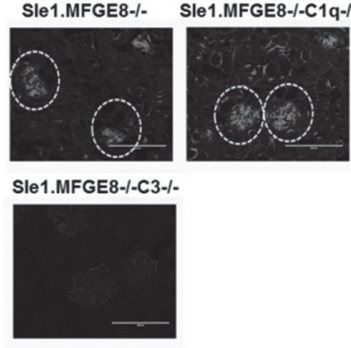

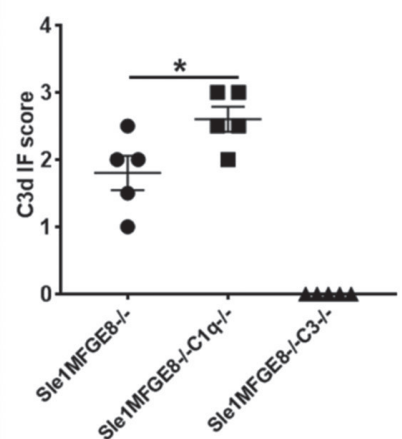

